\title{
Alignment and testing of critical interface fixtures for the James Webb Space Telescope
}

Kyle McLean ${ }^{\mathrm{a}}$, Paul Bagdanove ${ }^{\mathrm{a}}$, Joshua Berrier ${ }^{\mathrm{b}}$, Emmanuel Cofie ${ }^{\mathrm{c}}$, Tiffany Glassman ${ }^{\mathrm{d}}$, Theodore Hadjimichael $^{\mathrm{a}}$, Eric Johnson ${ }^{\mathrm{a}}$, Joshua Levi ${ }^{\mathrm{d}}$, Amy Lo ${ }^{\mathrm{d}}$, Joseph McMann ${ }^{\mathrm{e}}$, Raymond Ohl ${ }^{\mathrm{a}}$, Dean Osgood $^{\mathrm{e}}$, James Parker ${ }^{\mathrm{a}}$, Kevin Redman ${ }^{\mathrm{e}}$, Vicki Roberts ${ }^{\mathrm{e}}$, Matthew Stephens ${ }^{\mathrm{f}}$, Adam Sutton ${ }^{\mathrm{d}}$, Greg

Wenzele ${ }^{\mathrm{e}}$ Jerrod Young ${ }^{\mathrm{a}}$

${ }^{\mathrm{a} N A S A}$ Goddard Space Flight Center; ${ }^{\mathrm{b}} \mathrm{Tech}$ Innovations; ${ }^{\mathrm{c}}$ Stinger Ghaffarian Technologies Inc.;

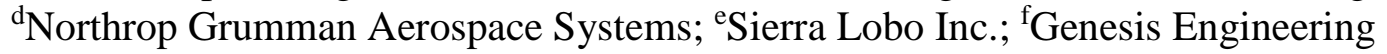

\begin{abstract}
NASA's James Webb Space Telescope (JWST) is a 6.5m diameter, segmented, deployable telescope for cryogenic IR space astronomy. The JWST Observatory architecture includes the Primary Mirror Backplane Support Structure (PMBSS) and Integrated Science Instrument Module (ISIM) Electronics Compartment (IEC) which is designed to integrate to the spacecraft bus via six cup/cone interfaces. Prior to integration to the spacecraft bus, the JWST observatory must undergo environmental testing, handling, and transportation. Multiple fixtures were developed to support these tasks including the vibration fixture and handling and integration fixture (HIF). This work reports on the development of the nominal alignment of the six interfaces and metrology operations performed for the JWST observatory to safely integrate them for successful environmental testing.
\end{abstract}

Keywords: JWST, Metrology, Optical alignment, Laser radar, Laser tracker, Theodolite, Coordinate measuring machine, CMM

\section{INTRODUCTION}

The James Webb Space Telescope (JWST) is a large infrared space telescope with a primary mirror of $\sim 6.5 \mathrm{~m}$ in diameter. The JWST is a NASA mission developed in conjunction with the European Space Agency and the Canadian Space Agency. The JWST observatory consists of two primary structures, the spacecraft bus, and the Optical Telescope Element (OTE) and Integrated Science Instrument Module (ISIM) (OTIS). The ISIM was integrated to the OTE (OTIS) in the fall of 2016. The OTIS interfaces to the spacecraft bus via six cup/cone interfaces, four Primary Mirror Back Support Structure (PMBSS), and two ISIM Electronics Compartment (IEC) interfaces.

The telescope must survive the unforgiving launch conditions and space environment, in addition to handling and transportation during the integration and test phase of the program. The OTIS structure undergoes environmental testing including vibration, acoustic, and thermal cycling to verify performance before and after the tests. To support these tests two Ground Support Equipment (GSE) fixtures, the OTIS Handling and Integration Fixture (HIF) (Figure 1-1), and the OTIS vibration fixture were developed, aligned, and tested. The HIF and vibration fixture support the various phases of the OTIS testing including vibration testing, acoustic testing, handling, and transportation. 


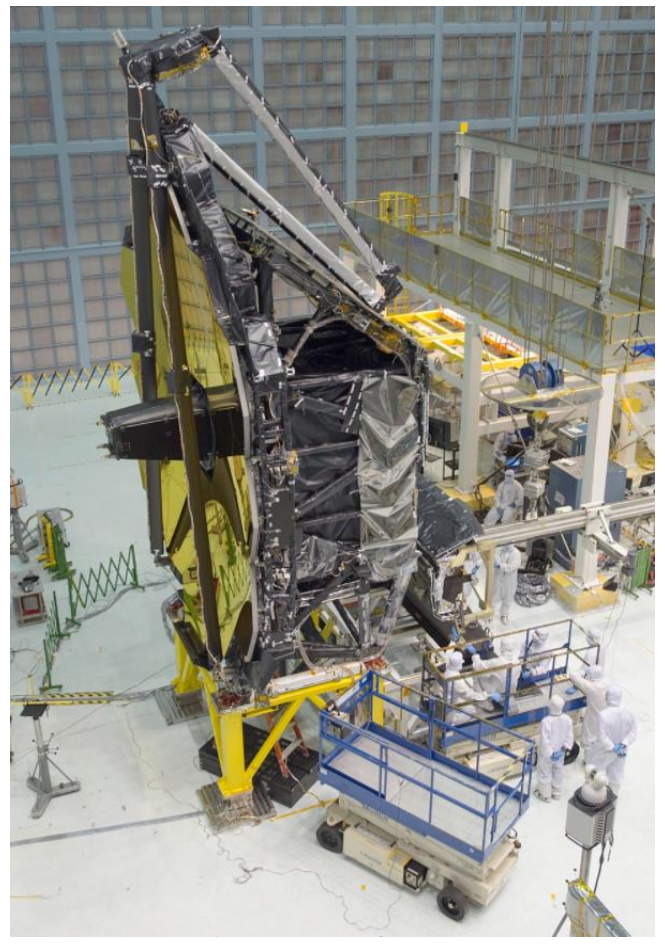

Figure 1-1 OTIS on the HIF for IEC integration at GSFC

The HIF was designed for the handling and transportation of the OTIS structure (Figure 1-2). In addition, the HIF is used for the IEC integration to the OTIS and defines the OTIS IEC cup interface locations. For IEC integration purposes, the IEC interfaces on the HIF are removable. A more robust structure was required to support the OTIS for vibration testing and acoustic testing. The vibration fixture required a monolithic base which interfaced to the vibration tables and the OTIS dolly (used to transport the OTIS on the vibration fixture to the vibration and acoustic facilities) to support the vibration testing (Figure 1-3).

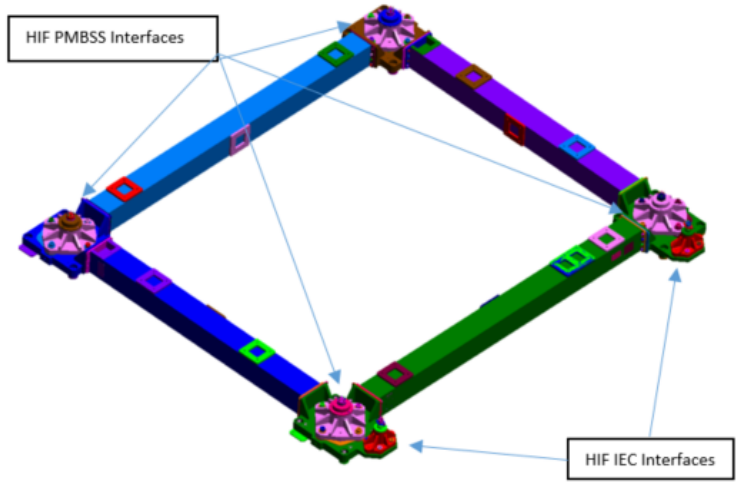

Figure 1-2 OTIS HIF 


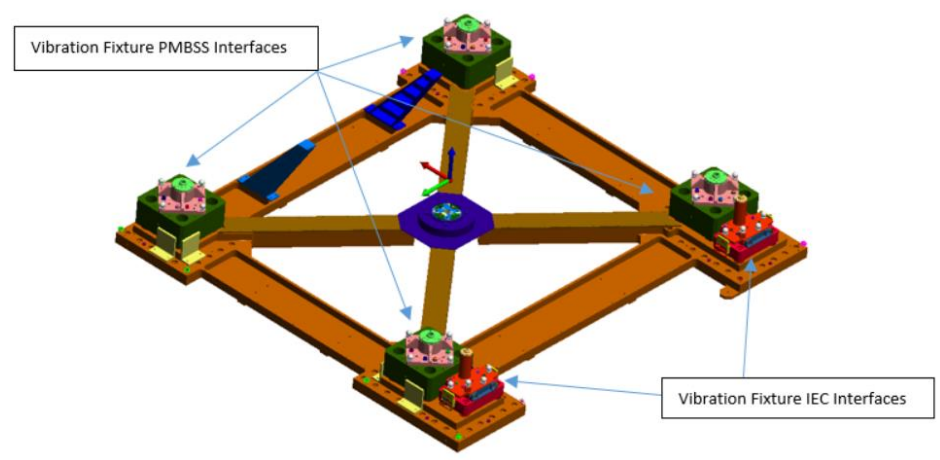

Figure 1-3 OTIS vibration fixture

Both fixtures are $\sim 2.6 \times 2.9 \mathrm{~m}$ and simulate the spacecraft bus cone interfaces that are used to support the OTIS during launch. The HIF and vibration fixtures six cone interfaces had to be aligned in both position and angle to a very tight requirement relative to the size of the fixtures (better than $0.125 \mathrm{~mm}$ in piston, $0.150 \mathrm{~mm}$ in translation, and 2 arc-min in rotation). The requirements were derived from structural analysis of the OTIS when subjected to vibration testing and transportation loads. The ridged non kinematic fixtures force large joint loading into the OTIS structure which dictated the small alignment requirement. The HIF and vibration fixture alignment requirement included factors such as OTIS interface knowledge, HIF/vibration fixture cone nominal misalignment, HIF/vibration fixture measurement uncertainty, thermal variations, different mechanical configurations and boundary conditions, and changes due to mechanical proof testing (Figure 1-4).

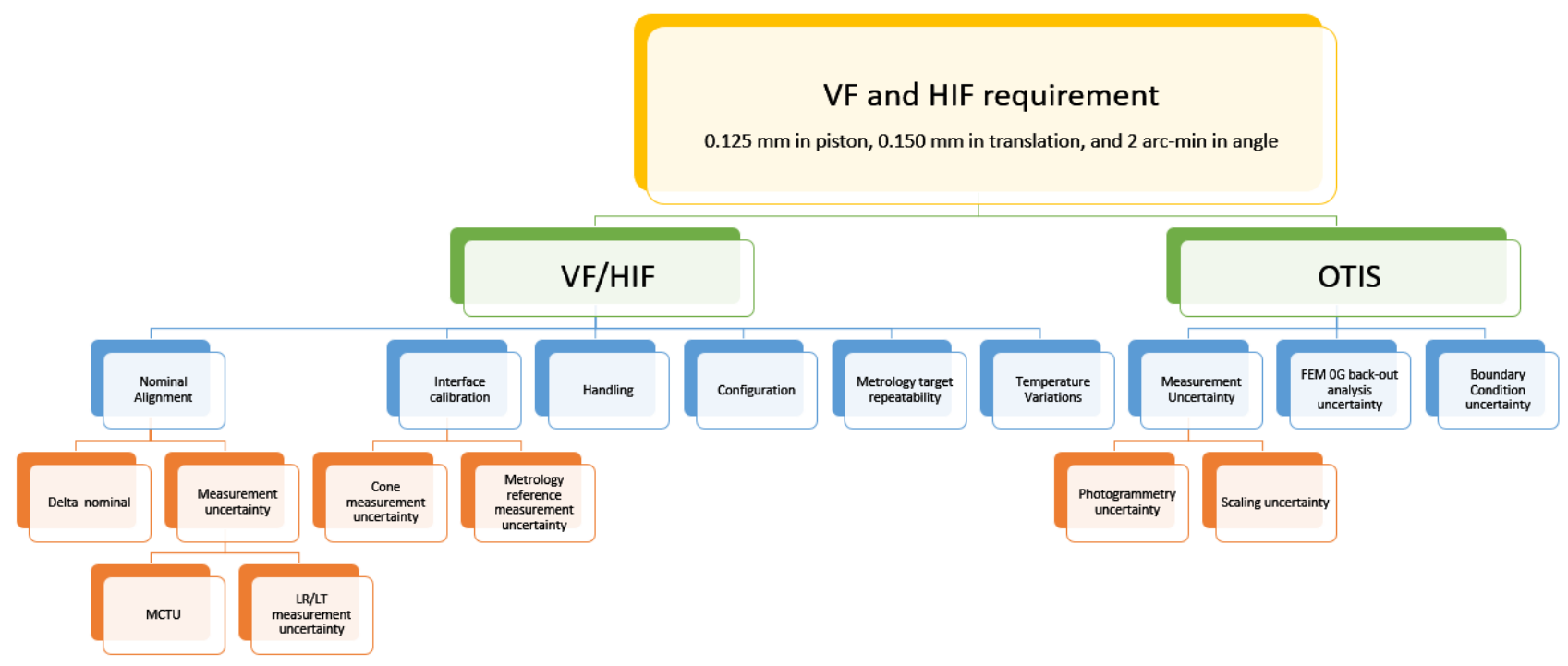

Figure 1-4 OTIS HIF and Vibration Fixture error budget

As previously noted, the HIF is used for the IEC integration and defines the relative alignment between the OTIS IEC cup interfaces and the OTIS PMBSS cup interfaces. The HIF IEC cone interfaces had to be positioned relative to the HIF PMBSS cone interfaces, such that the as-built OTIS minimizes the OTIS integration loads when mated to the spacecraft bus at the six locations (Upon receiving the OTIS, the six spacecraft cone interfaces had previously been aligned and pinned to the spacecraft bus). The OTIS PMBSS cup interfaces were measured and the deviation between the OTIS 
interfaces and the spacecraft interfaces were not acceptable for the vibration fixture and the HIF. The HIF and vibration fixture carried a much smaller alignment requirement than the spacecraft due to the additional stiffness of the two fixtures. As a result, the spacecraft interface alignment could not be directly utilized to define a nominal prescription for the HIF and vibration fixture. A nominal prescription was developed to minimize loads during vibration testing, handling, transportation, and the spacecraft launch.

\section{NOMINAL PRESCRIPTION DEVLOPMENT}

\subsection{OTIS PMBSS cup interface metrology}

Prior to the IEC integration and HIF alignment, an as-built configuration of the OTIS PMBSS interfaces was established to help define a nominal prescription for the HIF. The OTIS PMBSS cup interfaces were measured in two orientations, using two different metrology techniques to shed light on systematic uncertainties and provide a cross-check.

The primary method to define the OTIS PMBSS cup interface locations used photogrammetry, with the OTIS hanging from the crane. Photogrammetry targets were placed on the plane of the interfaces, and a spherical photogrammetry target was placed inside the cup interface. A local coordinate system was defined (OTE-SC) at the center of the four PMBSS interfaces. For consistency purposes, the measured interface angles and positions were analytically defined in a zero gravity state. This allows for comparisons to other measurements with different boundary conditions, and integration phases of the program.

Cross-check metrology tests were used to verify the photogrammetry results. The OTIS was positioned in the Ambient OTE Assembly Stand (AOAS). Metrology was performed with the primary mirrors facing up (cup up) and the primary mirror facing down (cup down). Two methods were used when measuring the PMBSS cup interfaces when in the AOAS. The first method was a direct measurement of the interface cup using a Laser Radar (LR) [1] and a "vision scan" (Figure 2-1). Each cup interface was scanned from multiple LR stations to capture the entire interface. Using Spatial Analyzer (SA) [2], a cone was best fit to the cup portion of the interface and a plane was best fit to the outer surface (Figure 2-1). The intersection of the cone center axis and the plane defines the center of the interface. All subsequent LR "vision scans" of the OTIS cups and HIF/vibration fixture cones were analyzed in a similar manner.

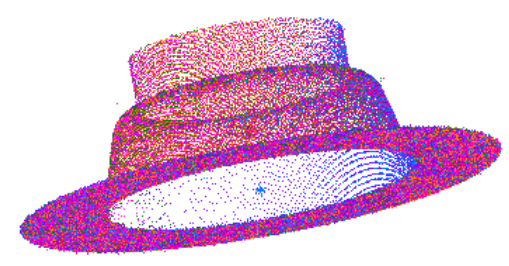

Figure 2-1 OTIS PMBSS cup interface LR scan

The second cross-check method placed a tooling ball in the cone for position (Figure 2-2), similar to the photogrametry positional technique, and parallel flats on the interface plane to measure the angles (Figure 2-3). The laser radar was used to measure the tooling balls with repsect to tie points. The tooling balls were removed and the optical flats added and measured using the LR and the "Direct and Through" technique [3]. The optical flats were calibratied prior to use to define the wedge angle between the measured optical surface and the datum which mates to the OTIS interfaces to correct for this error during analysis. The two LR methods (Direct scan, and tooling ball/optical flat) measuring the OTIS interfaces agreed within $0.020 \mathrm{~mm}$ in position and $30 \mathrm{arc}-\mathrm{sec}$ in angle. 


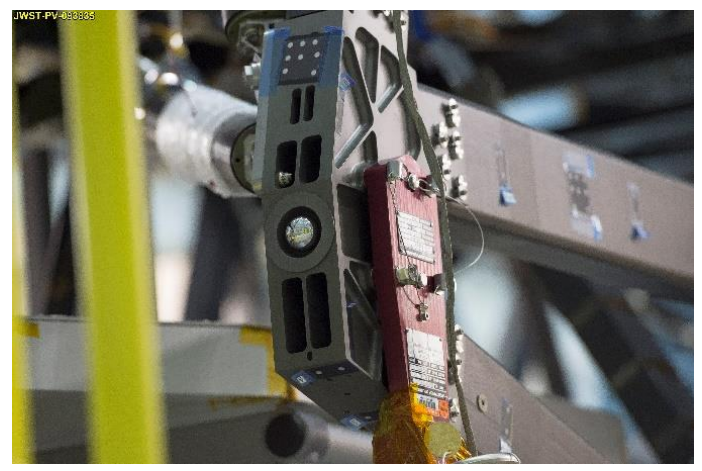

Figure 2-2 OTIS PMBSS Interface with tooling ball

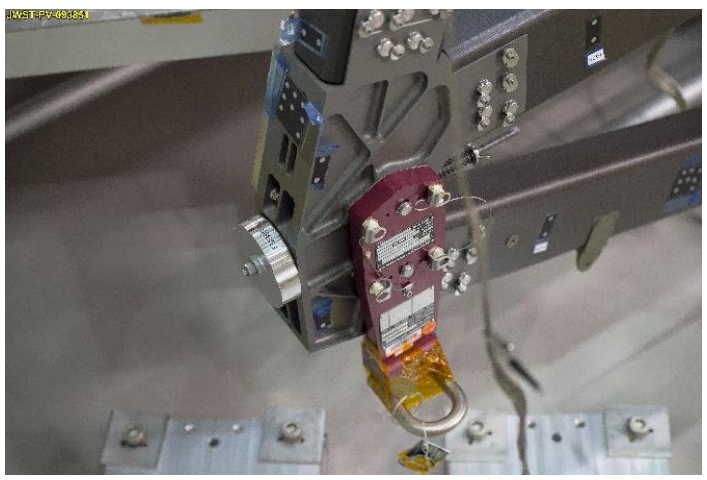

Figure 2-3 OTIS PMBSS Interface with optical flat

The LR interface metrology was backed out to the zero-gravity configuration through finite element modeling (FEM) analysis and compared to the photogrammetry results. The different measurement orientations and techniques agree within measurement and analysis uncertainty. The hanging/photogrammetry method was used as the primary metrology set due to the lower uncertainties associated with the boundary conditions and correction for the gravity effects.

\subsection{OTIS IEC cup interface metrology}

The OTIS IEC cup interfaces were measured prior to integration to the OTIS in order to help predict the interface locations. The IEC was positioned in the horizontal configuration, and the cones were scanned using the LR similar to the OTIS PMBSS interface direct scans. These scans were related to targets locally on each of the two interfaces, creating a calibration of the interface center and plane to metrology references near the interface (Figure 2-4). The calibration allowed for an indirect measurement of the IEC cups in different orientations via transformation. 


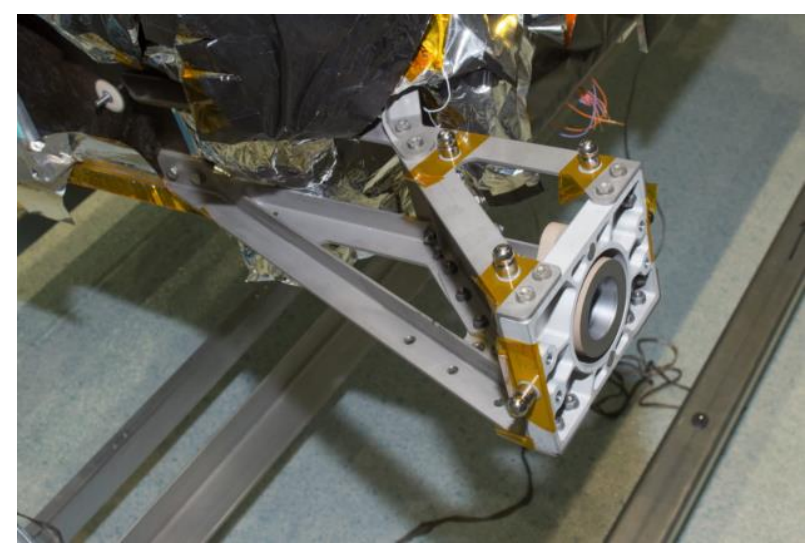

Figure 2-4 OTIS IEC cup with metrology reference nests and tooling balls

The IEC was then turned to the vertical configuration, similar to the integration boundary conditions, and the interface reference targets with tooling balls were measured using a LR. The interface calibrations from the horizontal configuration were transformed to the measured reference targets using a Monte Carlo Transformation Uncertainty (MCTU) code[4] developed at NASA Goddard Space Flight Center (GSFC) to propagate measurement uncertainties through ridged body transformation.

\subsection{HIF nominal prescription}

The six spacecraft cone interfaces were used as a template to define the nominal HIF cone interface locations. To minimize IEC loads due to integration, it was important to preserve the relative distance between the two -X PMBSS interfaces to the IEC interfaces. To accomplish this goal, the OTIS IEC cup interfaces were transformed to the spacecraft IEC cone interfaces using the two points, defining five degrees of freedom. This forced the two sets of interfaces to be centered and in line with one another. For the last degree of freedom (rotation about $\mathrm{Y}$ ) the individual interface angular errors were minimized. The OTIS PMBSS interfaces were transformed to the spacecraft using the $-X$ interfaces as the primary points similar to the IEC transformation, defining five degrees of freedom. The $+X$ PMBSS interfaces were used to define the rotation about Y. The OTIS interface positions after both the IEC and PMBSS interfaces were transformed to the spacecraft bus defined the nominal HIF locations and angles (Figure 2-5).

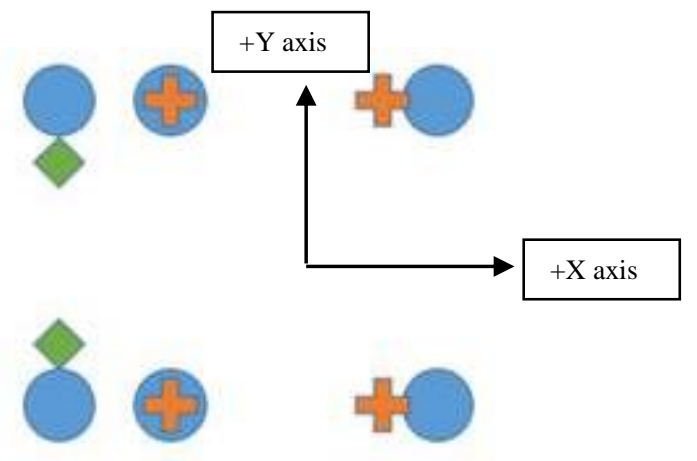

- SC Interfaces- Blue circles

- OTIS PMBSS Interfaces- Orange cross

- OTIS IEC Interfaces- Green diamond

Figure 2-5 Visual representation of the HIF nominal position definition 


\subsection{Vibration Fixture nominal prescription}

The HIF was aligned, and the as-built HIF locations and angles were defined. Because the HIF defines the relative alignment between the OTIS IEC and PMBSS cup interfaces, the vibration fixture nominal prescription was developed based on the HIF as-built alignment. The vibration fixture nominal interface locations were defined using a similar method used to define the HIF nominal interfaces. For the vibration fixture nominal prescription, instead of using the spacecraft interfaces as a template, the as-built HIF locations were used as the template.

An added complication that needed to be taken into account was that the vibration fixture warms up during vibration testing on the slip table (horizontal shaker) and the head expander (vertical shaker). The nominal operational temperature of the vibration fixture on the head expander and slip table is $\sim 24 \mathrm{C}$ during vibration testing, and the alignment temperature was $\sim 20 \mathrm{C}$. The nominal prescription of the HIF was adjusted using the coefficient of thermal expansion of the vibration fixture to account for this temperature difference to ensure proper alignment at the operational temperature.

\section{INTERFACE CALIBRATION}

\subsection{CMM calibration}

Each of the HIF and vibration fixture interfaces had a cone feature which was identical the mechanical spacecraft interfaces that mate to the OTIS, and were outfitted with tooling ball (TB)/Spherically mounted retro-reflector (SMR) metrology nests, and optical flats for alignment and verification (Figure 3-1). To reduce measurement errors, a contact probe Coordinate Measuring Machine (CMM) was used to calibrate the cone interface with respect to reference targets on the body of the cone interface adaptors. The CMM measured multiple points on each of the interface cones and planes, as well as TBs in the nests, and optical flats on each of the interfaces (Figure 3-2). Using geometry fitting a cone and plane was fit to each of the features. The intersection of the cone axis and the plane defines the position of the cone. This created an interface calibration of the cone relative to the metrology reference targets that allowed for an indirect measurement of the interfaces. The calibrations could be transformed into the coordinate system using the metrology nests and optical flats during the alignment and testing phase for the HIF and vibration fixture.

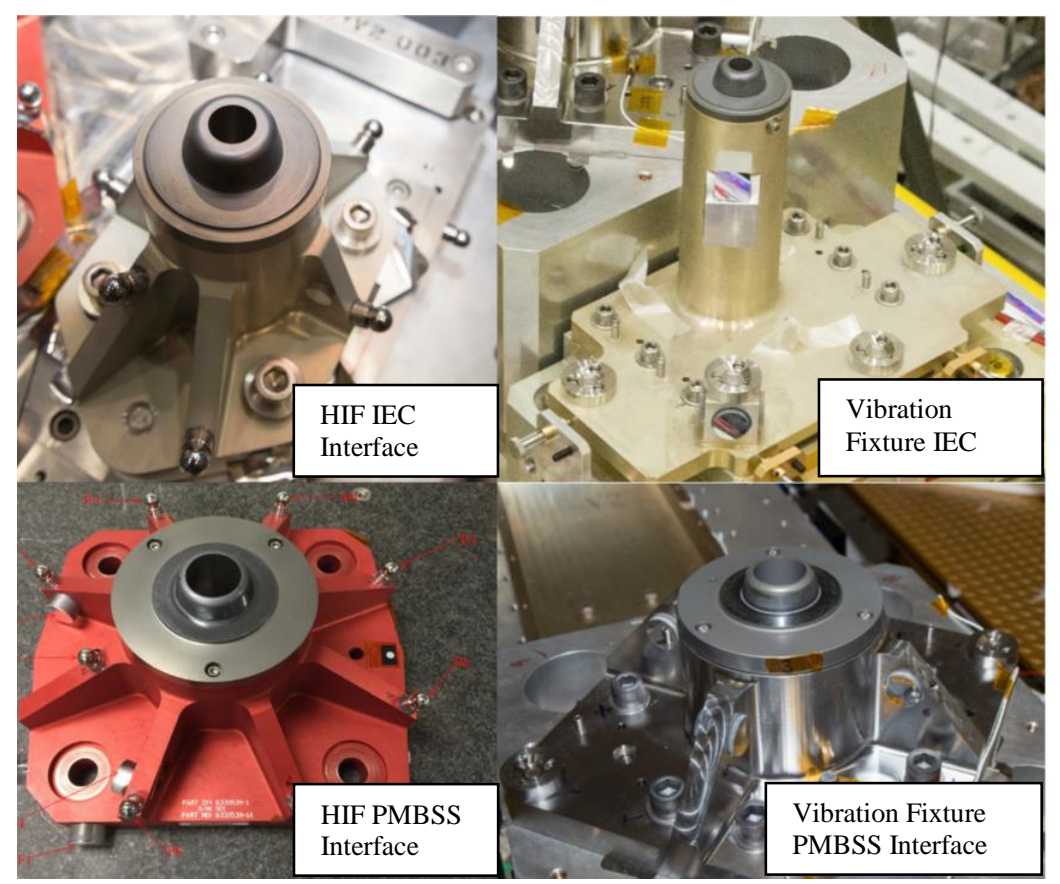

Figure 3-1 HIF and Vibration fixture interfaces 
The HIF IEC cones used smaller optical flats due to available real-estate on the interface. These smaller flats resulted in a higher angular measurement uncertainty when measured directly with the CMM. Auto-collimating theodolites were used to measure the optical flat in addition to a transfer cube assembly (TCA). The CMM also measured the TCA directly, which allows for the transformation of the theodolite results to the CMM measurements [5].

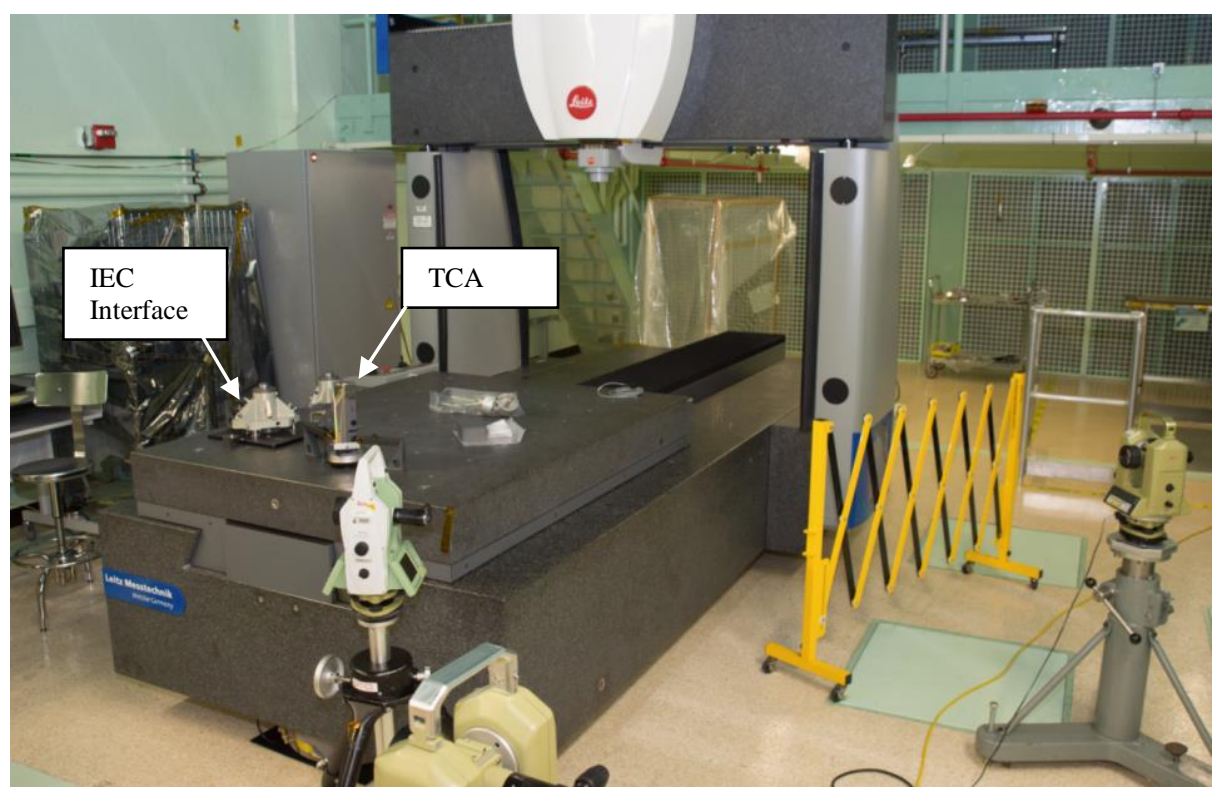

Figure 3-2 HIF IEC cone interface calibration on the Leitz PMM-C 700 Error! Reference source not found. with the TCA and interface mounted to the granite table

\subsection{LR cross-check}

A cross-check metrology was performed for each type of interface to verify the measurements of the CMM calibrations. A Laser Radar (LR) was used to measure the cones, metrology nests with tooling balls, and the optical flats. The cones were measured directly using a "vision scan" and the optical flats were measured using the "Direct and Through" technique. The (SA) Unified Spatial Metrology Network (USMN) [7] process was used to bundle the LR stations, and the measured nest locations were transformed to the CMM calibration. The transformation of the nests was compared, as well as the cone position and plane angle. The cross-check metrology showed an error in one of the interface calibrations. The probe diameter was properly not accounted for in one of the calibrations which resulted in a $2 \mathrm{~mm}$ error in the piston direction for the original CMM results. This error could have potentially led to a structural failure of the OTIS during testing and transportation. The independent cross-check demonstrates the importance of additional verification measurements to help mitigate risks for errors such as this.

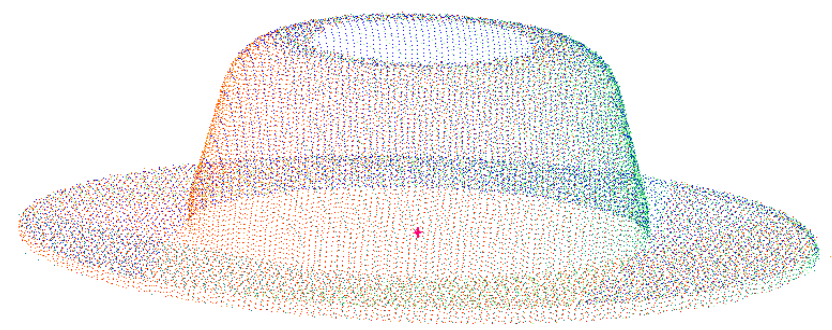

Figure 3-3 HIF PMBSS interface laser radar vision scan 


\section{ALIGNMENT}

\subsection{HIF Alignment}

The HIF was aligned on the IEC Integration Stand (IIS) using multiple LRs and a laser tracker (LT) [8] positioned around the fixture (Figure 4-1). Three LRs measured the metrology nests with tooling balls to align the interfaces. The SA USMN process was used to bundle the three stations. The interface calibrations described in the previous section were transformed to the UMSN group then compared to the nominal. The optical flats were measured with theodolites as a tool to initially align the interfaces in angle. The optical flat angles and the angles using the nests did not agree during the initial measurements. All of the optical flats were measured using theodolites to troubleshoot the discrepancy between the two measurements. The optical flats locally on an interface did not agree, indicating possible deformations locally within an interface. The nest targets are spread across the entire interface with a larger area, therefore are less susceptible to small local deformations. Therefore, only the nests were used to align and verify the angles of the interfaces instead of the optical flats.

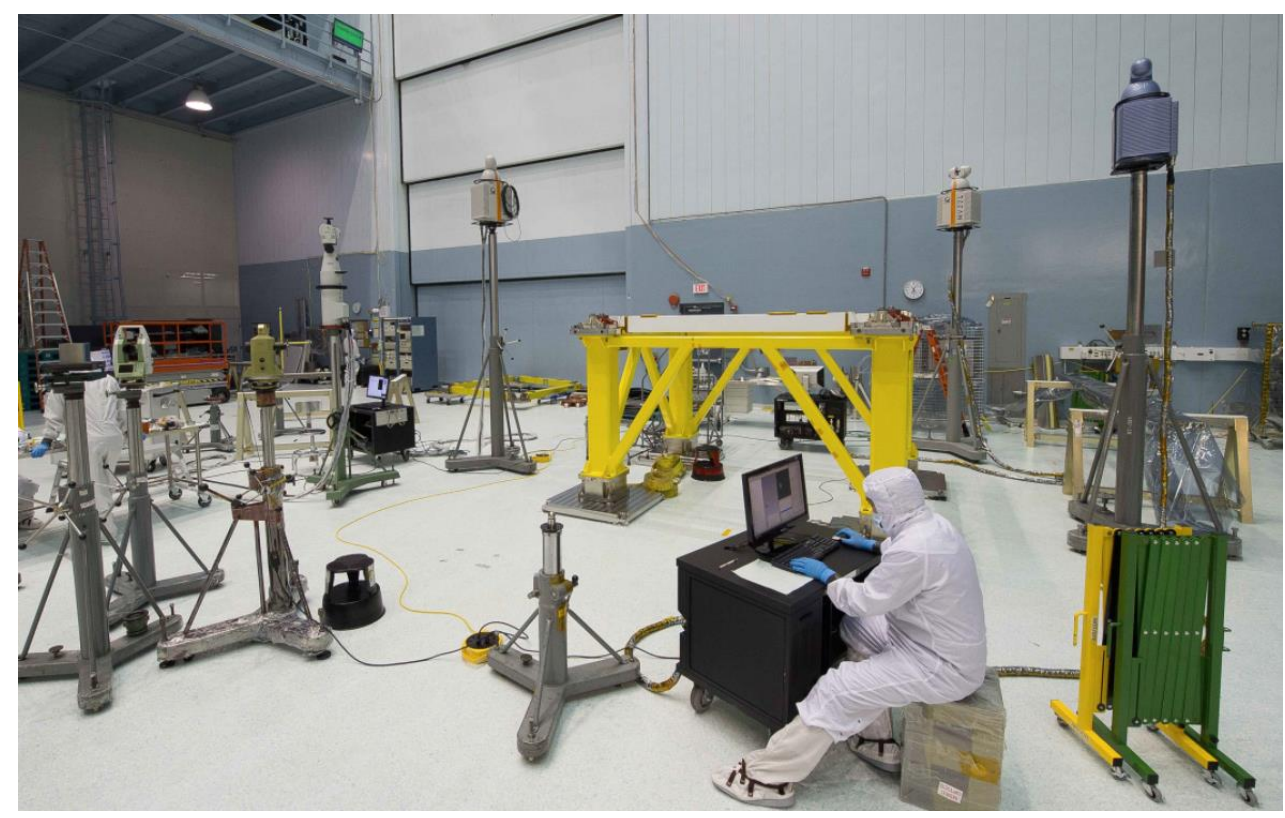

Figure 4-1 HIF alignment on the yellow IIS

The cone interfaces were first aligned in height and angle using shims between the cone interfaces and the HIF. To align the interface in translation, a Spherically Mounted Retro-reflector (SMR) was placed on top of the interface cone as a rough alignment using a "watch window." The LRs were used to characterize the actual location of the cone using the metrology nests, which created an actual distance to move to the nominal position. Through iterating between the LT and the LR measurements, the interfaces were aligned in translation.

Once the HIF cones were aligned in position and angle, a high fidelity metrology survey was completed to verify the HIF alignment. Each metrology nest on the interfaces were measured from a minimum of three LR positions. As a crosscheck, the cones were also measured directly using the LR "vision scan." The USMN analysis tool in SA was used to bundle the stations, and the interface calibrations were transformed to the USMN group using the MCTU code to define the position and angle of the interfaces with propagated uncertainties. The alignment of the HIF interface met the sub allocation of the requirement (Nominal misalignment sub allocation was $0.025 \mathrm{~mm}$ in piston, $0.040 \mathrm{~mm}$ in translation, and 30 arc-sec in angle). The laser radar cross-checks agreed with the primary analysis within $0.025 \mathrm{~mm}$ in position and 1 arc-min in rotation, which was well within the measurement uncertainties of the two measurement techniques. 


\subsection{Vibration Fixture Alignment}

The vibration fixture was aligned on the slip table which was used for the horizontal vibration testing of the OTIS (Figure 4-2). A LT was used to measure the reference targets with SMRs on the PMBSS interfaces in position, and theodolites were used to align the interfaces in angle. The theodolites were transformed into the coordinate system defined by the LT measurements using a Transfer Cube Assembly (TCA) and "direct and through" technique. Similar to the HIF alignment the interface calibrations were transformed to the measured metrology nests to define the interface position.

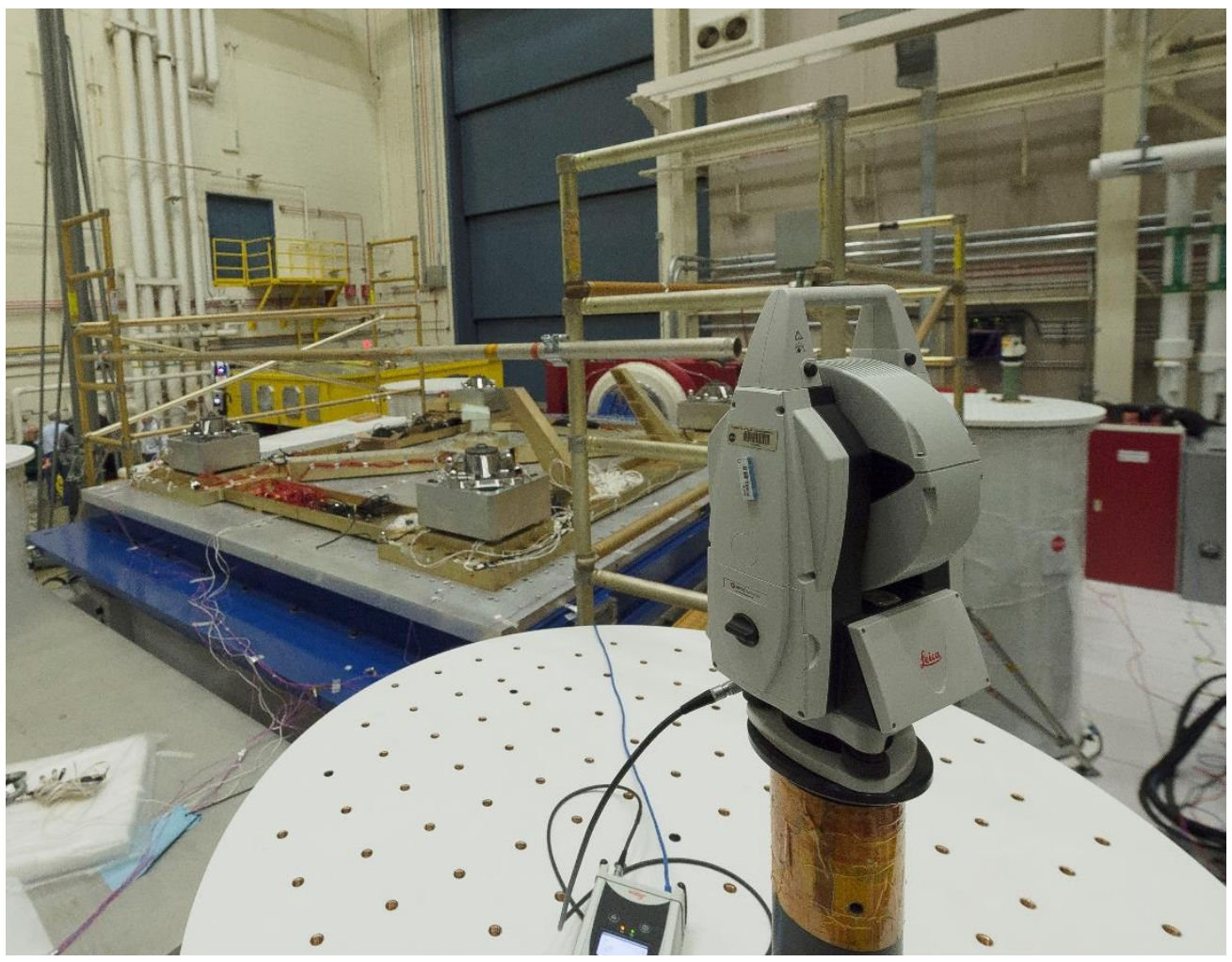

Figure 4-2 Vibration fixture on slip table during alignment

When aligning the HIF IEC cones, the metrology nests were measured and significant distortion of the nests were observed when the calibration was transformed to the measurement. The interface was measured both torqued and with the fasteners released, and it was found that the distortion was a result local deformations from the load applied once torqued to the vibration fixture. The distortion of the interfaces did not allow for use of the metrology nests on the IEC cone interfaces to relate the cone position. The interface angle was verified using theodolites and the optical cube on the post for the cone interface which was not affected by the distortions at the base. Laser radars were positioned around the IEC interfaces, and measured the cone positions directly using a vision scan. A delta nominal was found, then using a LT "watch window" the interface was translated. The iterative process was repeated multiple times until the cone was positioned within the sub allocation requirement. The angles were measured and aligned using the cube on the "post" of the HIF IEC cone interface.

Once the interfaces were aligned in position and angle, a high fidelity metrology survey was completed to verify the vibration fixture alignment. Each metrology nest on the interfaces were measured from a minimum of three LT/LR positions, and all optical flats were measured using theodolites. The cones were also scanned directly from multiple stations using a LR. The cone scans were used as a cross-check for the vibration fixture PMBSS cone interfaces, and the primary analysis tool for the IEC cone interface position. The USMN analysis tool in SA was used to bundle the stations, and the interface calibrations were transformed to the USMN group using the MCTU code to define the position and 
angle of the interfaces. The theodolite measurements were used as a cross-check of the transformed interface angles from the metrology nests on the vibration fixture PMBSS cone interfaces, and the primary analysis for the IEC cone interfaces. The alignment of the vibration fixture interfaces et the sub allocation of the requirement (Nominal misalignment sub allocation was $0.025 \mathrm{~mm}$ in piston, $0.040 \mathrm{~mm}$ in translation, and $30 \mathrm{arc}-\mathrm{sec}$ in angle). The theodolite and LT angular measurements agreed within 15 arc-sec, and the vibration fixture PMBSS cone interface scans and transformed locations agreed within $0.030 \mathrm{~mm}$, which was below the measurement uncertainty for the two techniques. With good agreement between the two measurements, all subsequent tests only utilized the metrology nests to determine if the interfaces shifted.

\section{ALIGNMENT VERIFCATION}

\subsection{HIF Alignment Verification}

The HIF is used on other structures and configurations through the integration and testing phase of the OTIS. Multiple alignment checks were completed to insure proper alignment of the HIF to capture errors associated with these configurations. All HIF measurements were completed in a similar manner, measuring all targets from a minimum of three LR and/or LT positions. Followed by a UMSN to bundle the stations and transforming the interface calibrations using the MCTU code.

After the interface alignment, the HIF was pinned and subjected to static load proof testing to ensure the HIF can survive the forces of OTIS during transportation (Figure 5-1). After the proof test the HIF was moved back to the IIS and the alignment was verified. It was observed the interfaces had moved $\sim 0.150 \mathrm{~mm}$ relative to the HIF base due to the loads subjected during proof testing. The shift between the HIF and cone interfaces was a result of the shear forces breaking the friction between the two parts. Once the load shear load was removed from the interfaces some of the misalignment induced was held in place by friction. The fasteners holding the interfaces to the HIF were released and re-torqued to the HIF, and the interface positions improved near the original alignment. The pins likely held the position of the interfaces with minimal plastic deformation. While no physical damage was observed to the HIF and was sufficient from a structural failure stand point, the HIF failed the proof test as a result of the apparent movement of the interfaces. These measurements demonstrate importance of metrology before and after, where a part may pass from a structural failure perspective, but the critical alignment may be modified which would not have been captured without the metrology tests. Additional and larger pins were added to mitigate this problem, and the alignment held after the proof test was repeated.

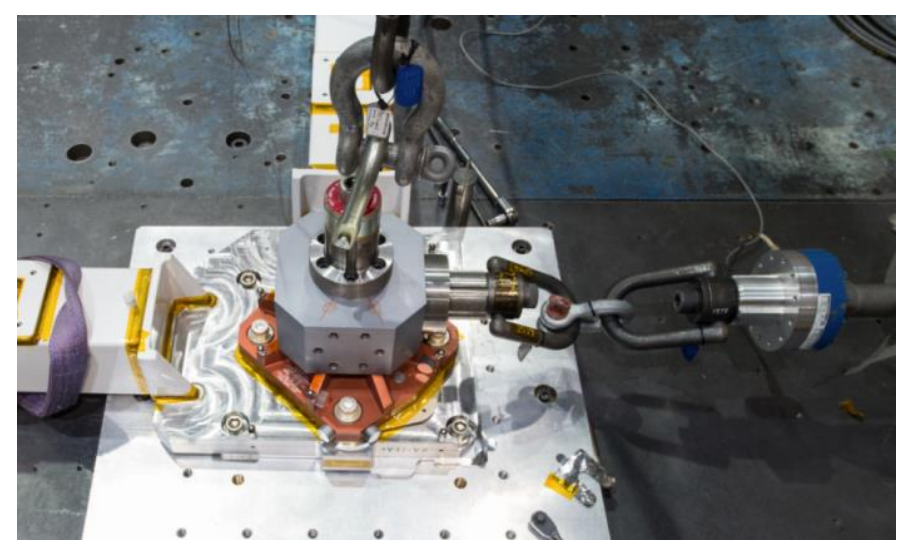

Figure 5-1 HIF static load proof test

After proof testing the HIF was ready for OTIS use. The HIF alignment during integration of the OTIS was important to ensure any misalignment was not locked into the system once integrated. The OTIS was first integrated to the HIF on the High Capacity Roll Over Fixture (HCROF) (Figure 5-2). Due to different surface profiles of the IIS and the HCROF, shims had to be added between the HIF and the mounting surface to insure proper alignment. This process was repeated with the HIF placed onto the Space Telescope Transportation Air Road and Sea (STTARS) shipping container prior to the OTIS integration for transportation (Figure 5-2). 


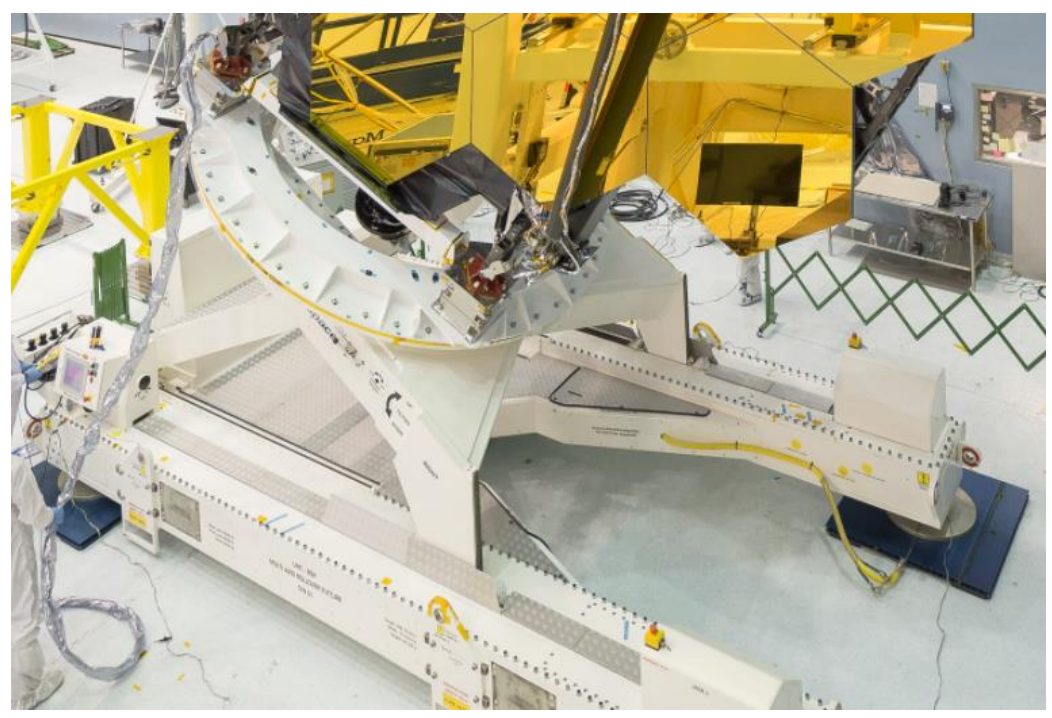

Figure 5-2 HCROF with OTIS and HIF

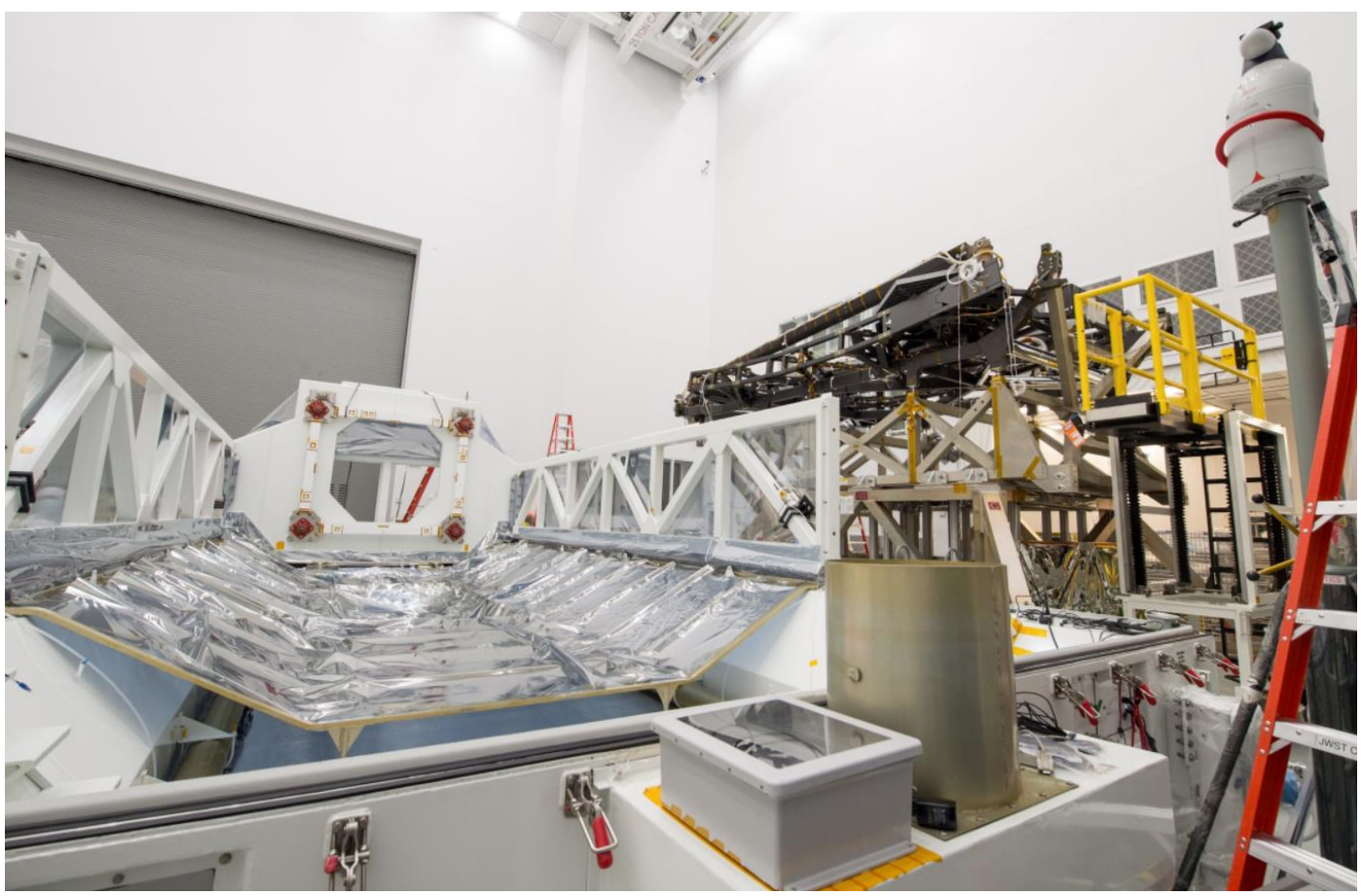

Figure 5-3 HIF on STTARS

Another factor affecting the alignment of the HIF when on the STTARS, is distortion of the container during transportation. A displacement on one end of the STTARS can cause distortion at the STTARS HIF mating surfaces. A metrology test was completed to characterize these affects and were correlated to a mechanical Finite Element Model (FEM). Metrology targets were positioned at various points on the STTARS (Figure 5-4) including 16 points of the HIF mounting datum (Figure 5-5) on the container. Using a LT, displacements were applied using leveling jacks (Figure 5-6) at the corners of the STTARS, then a metrology survey was completed. This test was completed with different magnitudes of displacements at different corners, both with the lid on and the lid off (Figure 5-7 and Figure 5-8). 


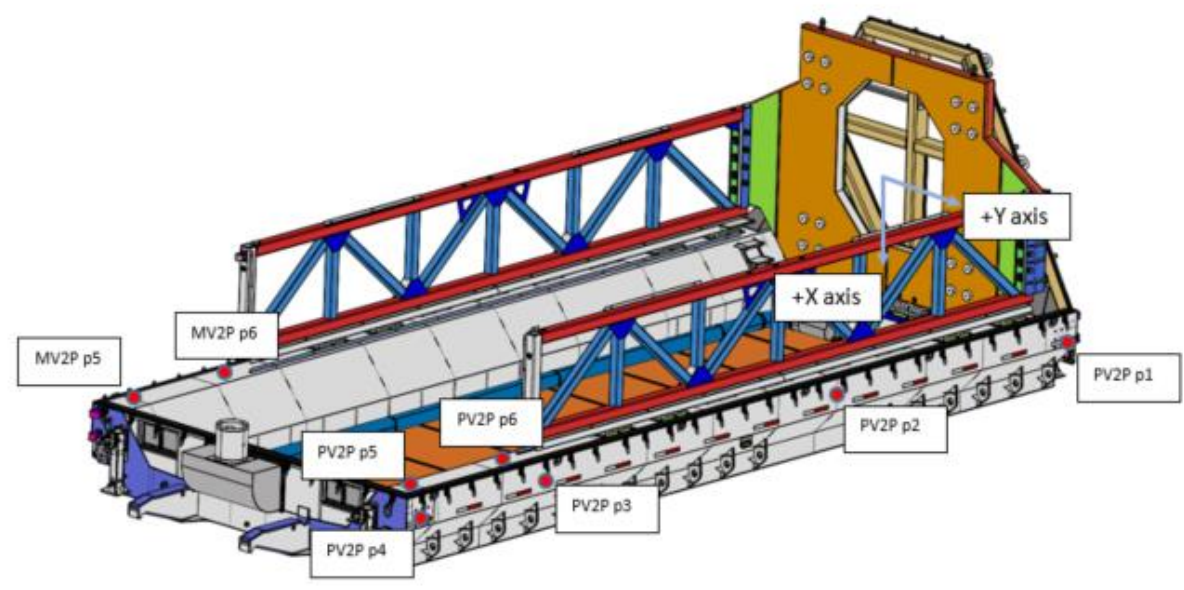

Figure 5-4 Metrology target locations on STTARS during distortion testing

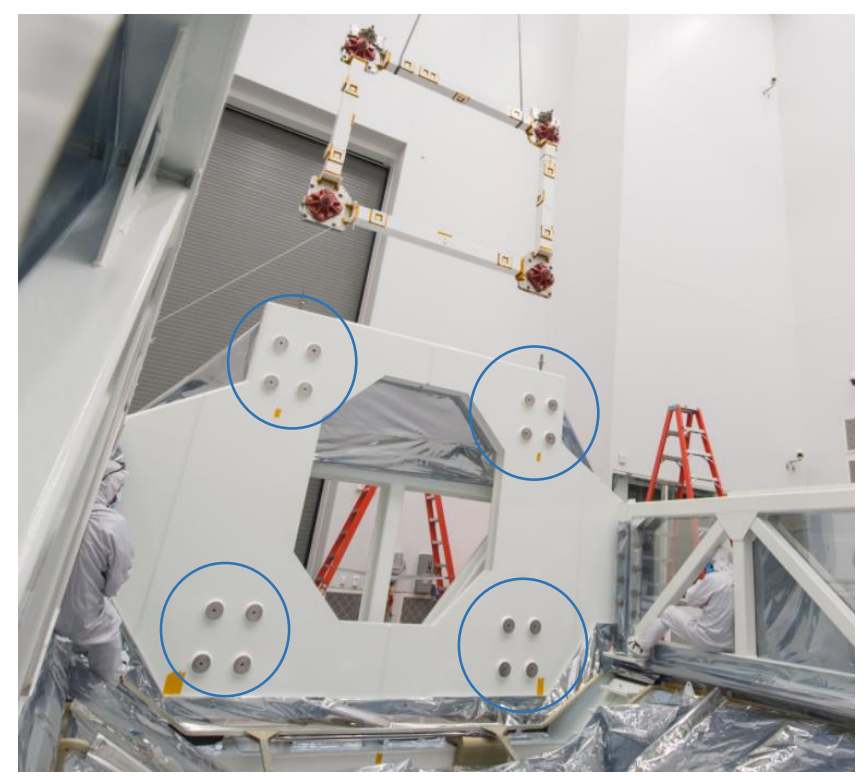

Figure 5-5 STTARS HIF interface locations (16 bolt holes) 


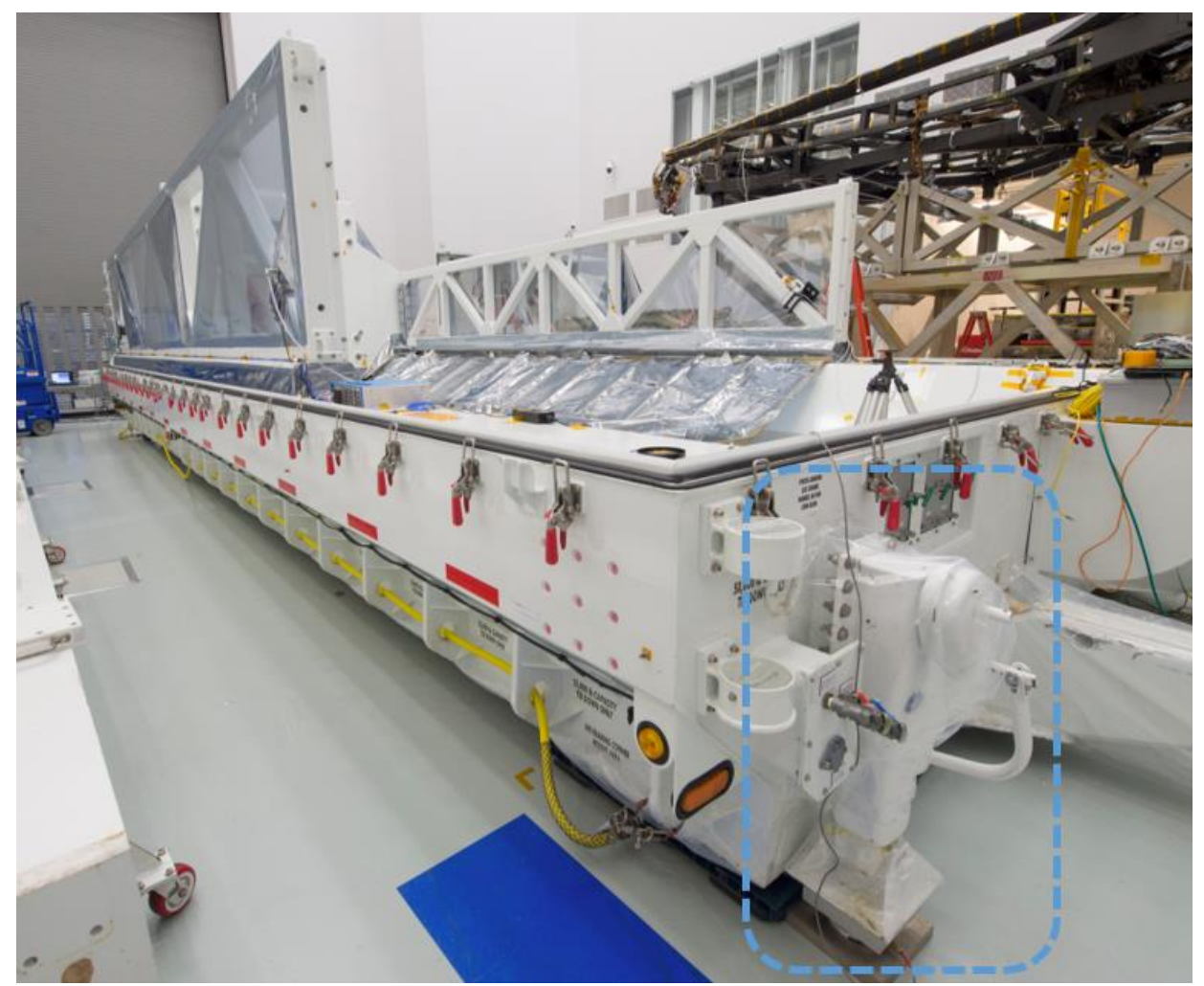

Figure 5-6 STTARS leveling jack

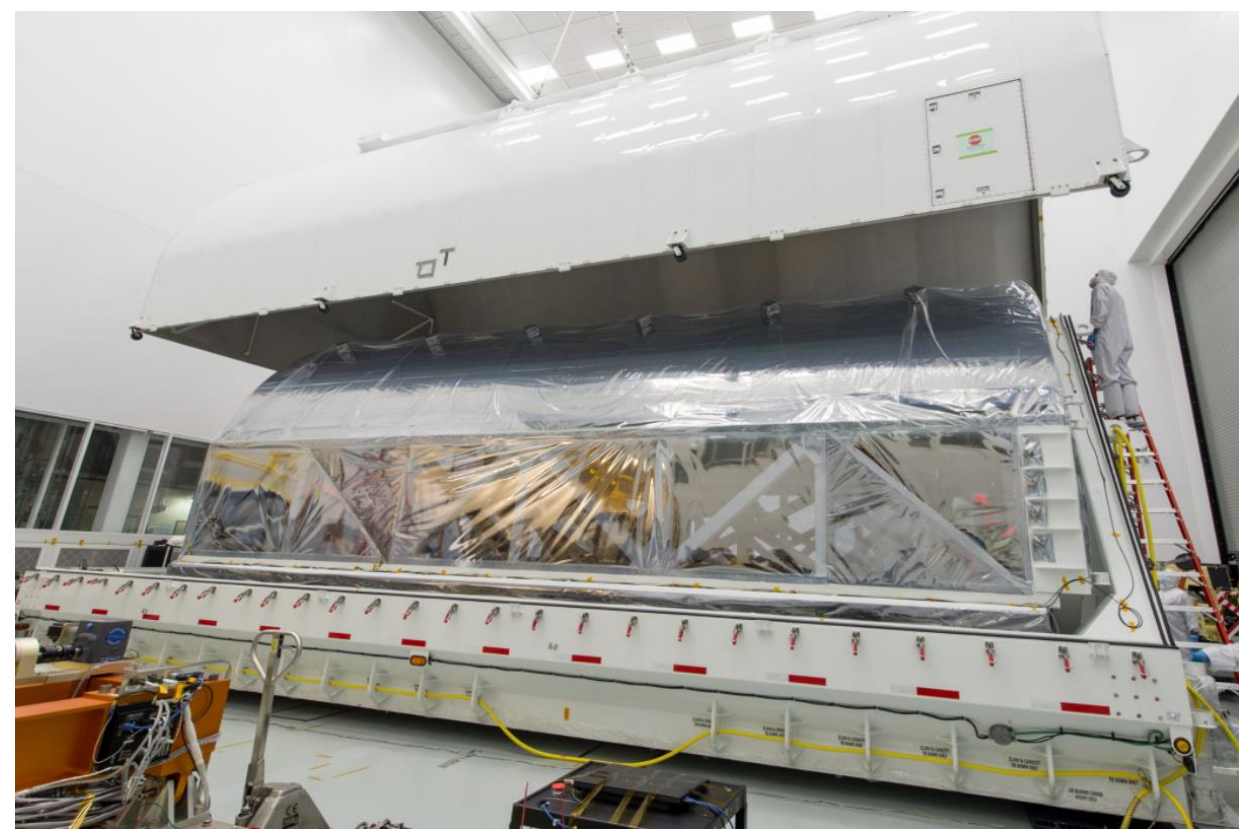

Figure 5-7 Lid integration to STTARS 


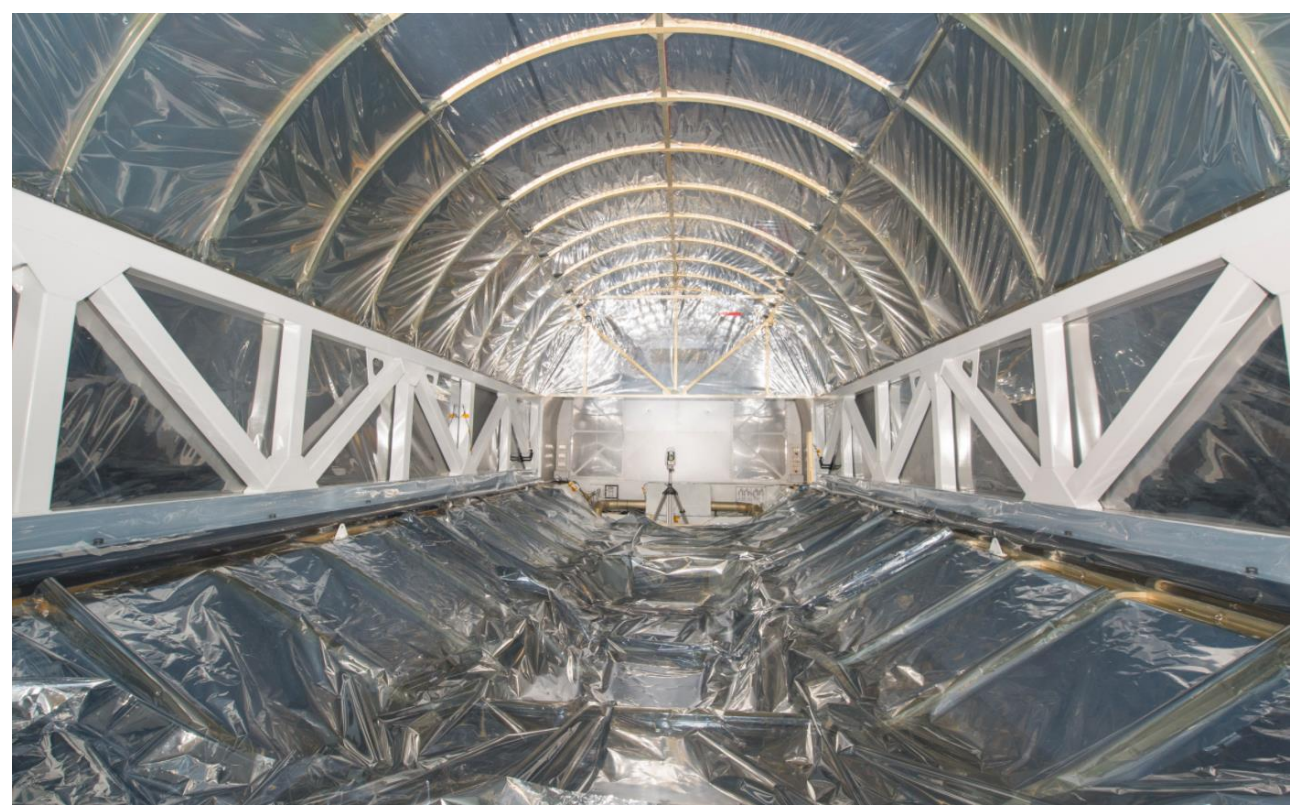

Figure 5-8 Leica AT401 positioned in the STTARS with the lid on, measuring the STTARS HIF interfaces (View from the HIF interface end of STTARS)

\subsection{Vibration Fixture Alignment Verification}

Similar to the HIF, the vibration fixture is used on other structures and configurations through the integration and testing phase of the OTIS. The same HIF metrology and analysis techniques were used to verify the vibration fixtures alignment for the different configurations.

As previously noted the vibration fixture was aligned at a lower temperature than the operational temperature. The vibration fixture was warmed up to the $24 \mathrm{C}$ and measured in two different orientations. The interface alignment relative to the nominal was within the sub allocation designated to this configuration (Nominal misalignment sub allocation was $0.025 \mathrm{~mm}$ in piston, $0.040 \mathrm{~mm}$ in translation, and 30 arc-sec in angle). The vibration fixture was then positioned onto the head expander and a similar test was completed. While the slip table heats uniformly, the head expander has heat inputs to only two sides resulting in a temperature gradient when in use (Figure 5-9). The objective of the head expander metrology was to correlate the thermal model as the head expander temperature increases. The alignment deviations due to thermal variations on the head expander were accounted for in another error budget, and not tracked in the metrology error budget.

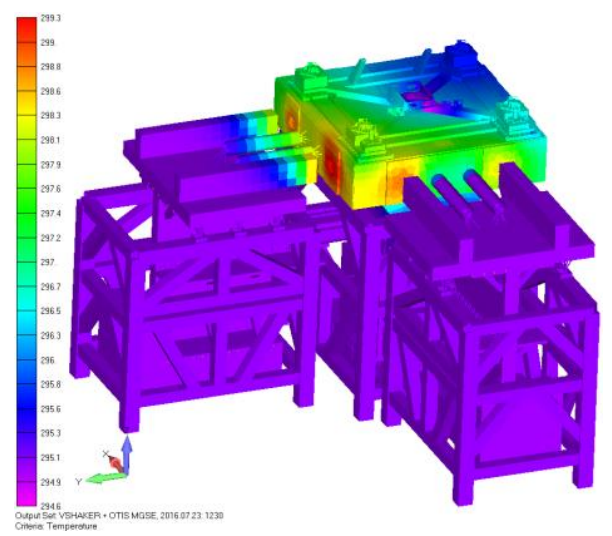

Figure 5-9 Vibration fixture on the head expander thermal model 
The vibration fixture, slip table, and head expander underwent proof testing using a mass simulator that interfaced with the vibration fixture PMBSS cone interfaces (Figure 5-10). Measurements were completed before and after each axis to insure the vibration fixture remained aligned.

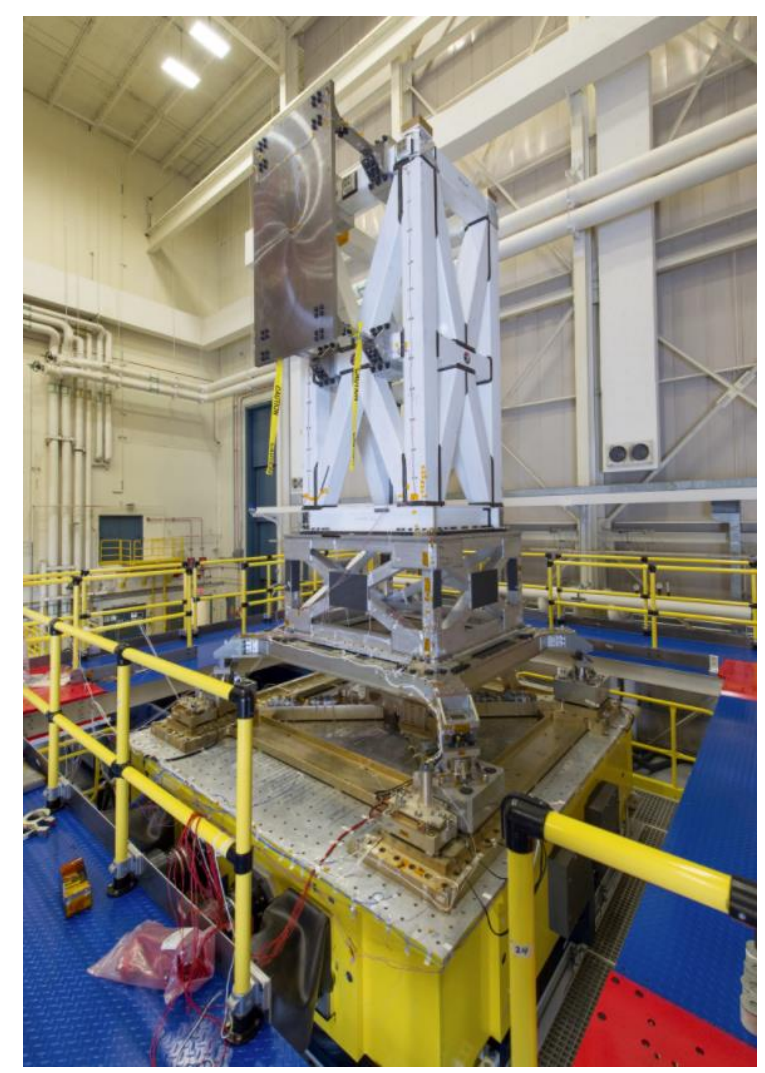

Figure 5-10 Vibration fixture with mass simulator on head expander for proof testing

To ensure proper alignment during OTIS integration the vibration fixture was measured when on the OTIS dolly. The dolly was used to transport OTIS at GSFC between the SSDIF, vibration testing, and acoustic testing. For repeatability purposes the dolly was leveled using three jacks. Shims were added below the vibration fixture to properly align the fixture. Prior to the OTIS integration to the vibration fixture, the SSDIF temperature was increased to 24C, which allowed for the vibration fixture to expand to the nominal position which decreased the integration loads (Figure 5-11). 


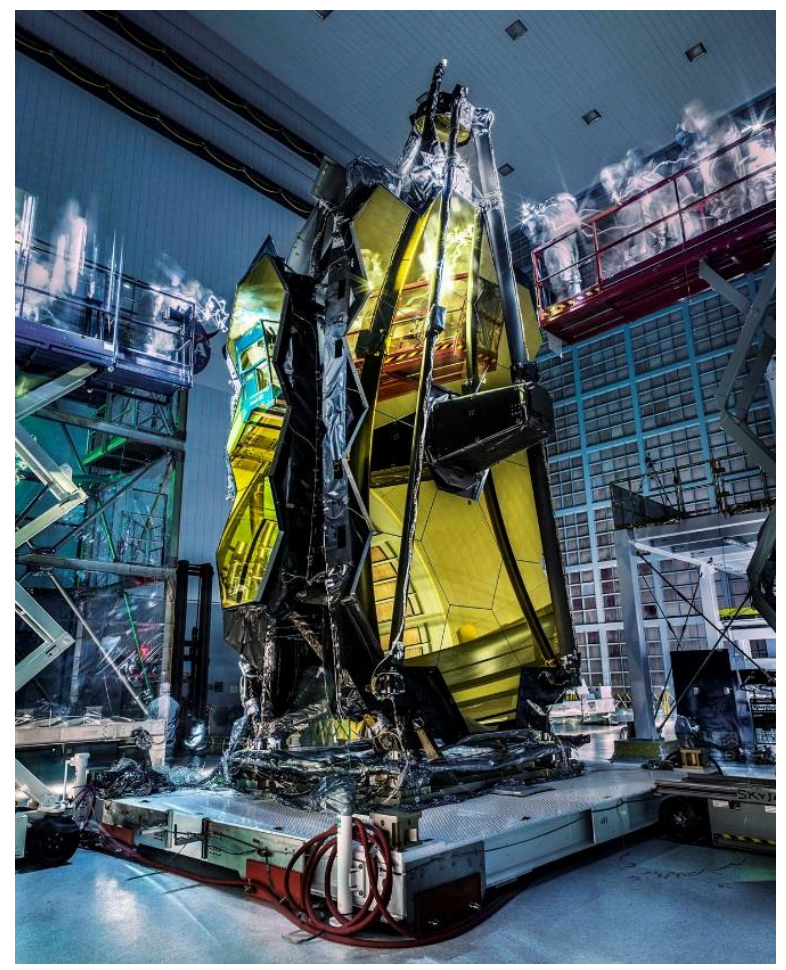

Figure 5-11 Vibration fixture with OTIS on the dolly

\section{CONCLUSION}

This paper has provided an overview of the alignment, testing, and verification processes used at the NASA GSFC in preparation of the HIF and vibration fixture for OTIS use. The OTIS successfully completed both vibration and acoustic testing on the vibration fixture, and the OTIS was successfully transported in STTARS to Johnson Space Center on the HIF for cryogenic testing prior to integration with the spacecraft and flight.

\section{REFERENCES}

[1] A. Slotwinski and P. Blanckaert, "Frequency Modulated Coherent Laser Radar Technology," Proceedings of the OPTIMESS2007 Workshop, Leuven, Belgium, May 28-30, 2007.

[2] New River Kinematics, Inc., Williamsburg, Va.

[3] Spatial Analyzer Users Manual, New River Kinematics, Williamsburg, v. 1.21.2008, page 150.

[4] J. Hayden, M. Khreishi, T. Hadjimichael, and R. Ohl, Monte Carlo Method for Uncertainty Propagation in JWST Metrology Databases, Coordinate Metrology Systems Conference, 2014.

[5] C. Aviado, J. Gill, K. Redman, and R. Ohl, Methods for correlating autocollimation of theodolites and coordinate metrology in spacecraft systems, Proc. SPIE 6273, 62733H, 2006

[6] Hexagon Metrology Inc., 250 Circuit Dr., North Kingstown, RI.

[7] S. Sandwith and R. Predmore, "Real-time 5-Micron Uncertainty with Laser Tracking Interferometer Systems using Weighted Trilateration," Proc. SPIE.

[8] J. Burge, P. Su, C. Zhao, and T. Zobrist, "Use of a commercial laser tracker for optical alignment," Optical System Alignment and Tolerancing, J. Sasian and M. Ruda, eds., Proc. SPIE 6676, SPIE Press, Bellingham, 66760E-1—12, 2007 